\title{
Über die \\ Ramanujan-Entwicklung multiplikativer Funktionen
}

von

WOLFGaNG SctrWarz (Frankfurt am Main)

1. Einleitung. Eis bezeichne

$$
c_{q}(n)=\sum_{d\{(q, n)} d \cdot \mu\left(\frac{q}{d}\right)=\sum_{\substack{a=1 \\(a, q)=1}}^{q} \exp \left(2 \pi i \frac{a}{q} \cdot n\right)
$$

die Ramanujan-Summe (man vgl. [7]),

$$
M(f)=\lim _{x \rightarrow \infty} \frac{1}{x} \cdot \sum_{n \leqslant x} f(n)
$$

den Mittelwert dei zahlentheoretischen Funktion $f$ (falls dieser existiert), tund.

$$
\mathscr{F}_{0}=\{f: N \rightarrow C ;|f| \leqslant 1, M(f) \neq 0, f \text { multiplitaativ }\}
$$

die Menge der komplexwertigen multiplikativen Funktionen vom Betrage $\leqslant 1$, die einen nichtversehwindenden Mittelwert besitzen.

In einer früheren Arbeit [11] zeigte der Verfasser mit Methoden von G. Halász ([4], [5]), daßB stark multiplikative Funktionen $\left({ }^{1}\right)$ aus $\mathscr{F}_{0}$ eine punktweise konvergente Ramanujan-Entwickluwg

$$
f(n)=\sum_{q=1}^{\infty} a_{q} c_{q}(n), \quad n=1,2, \ldots
$$

besitzen. In [8], [9] wurden Ramanujan-Entwicklungen reellwertiger Funktionen aus $\mathscr{F}_{0}$ untersucht, was sich als wesentlich einfacher erwies, da sich diese Entwicklungen als absolutikonvergent herausstellen.

In der vorliegenden Note sollen die Ergebnisse aus [11] auf beliebige Funktionen aus $\mathscr{F}_{0}$ ausgedelnt werden (Satz 1). Ein nützliches Hilfsmittel hierbei ist ein einfaches Eirgebnis (Satz 3) über „benachbarte" multipli-

(1) d.h. es ist $f(n)=\prod_{p \mid \eta} f(p)$. 
kative Funktionen, das zu einem Ergebnis von Delange analog ist. Schließlich wird (Satz 2) in $\$ 7$ die Parseval'sche Gleichung für Funktionen aus Fo hergeleitet.

2. Ergebnisse. In [9] wurden die „Fourierkoeffizienten" $\left({ }^{2}\right) a_{q}(f)$ für reellwertige Funktionen $f_{\epsilon} \mathscr{F}_{0}$ bestimmt; es ist naheliegend, für beliebige $f \in \bar{F}_{0}$ dieselben Fourierkoeffizienten zu erwarten.

Wir definieren zunächst die multiplikative Funktion $h: N \rightarrow C$ durch

$$
h\left(p^{r}\right)=\left\{\begin{array}{lll}
0 & \text { für } & r=1, \\
f\left(p^{r}\right)-f(p) \cdot f\left(p^{r-1}\right) & \text { für } & r \geqslant 2,
\end{array}\right.
$$

und setzen zur Abkürzung für Primzahlen $p$

$$
\eta(p)=1+\sum_{r=2}^{\infty} p^{-r} \cdot h\left(p^{r}\right)
$$

Bemerkung. Auf Grund eines Satzes von Delange (man vgl. \$ 4 , Lemma la) gibt es ein $r \geqslant 1$, so daß $f\left(2^{r}\right) \neq-1$ ist; dies hat zur Folge, $\mathrm{da} \beta \quad \eta(2) \neq 0$ ist; $\eta(p) \neq 0$ für $p>2$ ist offensichtlich.

Dann definieren wir eine multiplikative Funktion $q \rightarrow a_{q}^{*}$ durch ihre Werte an den Primzahlpotenzen, nämlich

(2.3) $\quad \eta(p) \cdot a_{j p^{r^{r}}}^{*}$

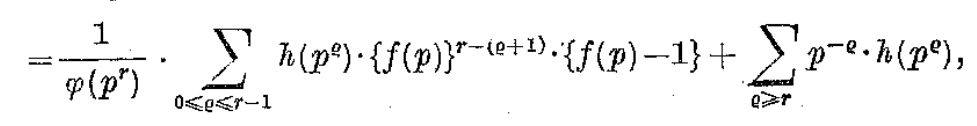

und setzen

(2.4)

$$
a_{q}=M(f) \cdot a_{q}^{*}
$$

Wir zeigen

SATZ 1. Sei $f \epsilon_{F_{0}}$; mit den durch (2.3) und (2.4) definierten Koeffizienten konvergiert die Reihe $\left(^{3}\right)$

$$
\sum_{q=1}^{\infty} a_{q} \cdot c_{q}(n)
$$

fiir $n=1,2, \ldots$ gegen den Wert $f(n)$.

SATz 2. (Parseval'sche Gleichung). Für $f \in \mathscr{F}_{0}$. lonvergiert die Reihe

$$
\sum_{q=1}^{\infty}\left|a_{q}\right|^{2} \cdot \varphi(q)=M\left(|f|^{2}\right)
$$

gegen den Mitelwert von $|f|^{2}$.

(2) Statt $a_{q}(f)$ schreiben wir in Zukunft der Kürze halber nur $a_{q}$.

$\left.{ }^{3}\right)$ Es kann nicht erwartet werden, daß die Konvergenz absolnt oder gleichmäßig in $n$ ist.
FOLGERUNG ZU SATZ 2. Fïr $f \in \mathscr{F}_{0}, g \in \mathscr{F}_{0}$ gilt $\left({ }^{4}\right)$

$$
\sum_{q=1}^{\infty} a_{q}(f) \cdot \overline{a_{q}(g)} \cdot q(q)=I I(f \cdot \bar{g})
$$

3. Benachbarte multiplikative Funktionen. Wir nennen zwei multiplikative Funktionen $f, g$ benachbart, wenn die über die Primzahlen erstreckto Summe

$$
\sum_{p} p^{-1} \cdot|f(p)-g(p)|<\infty
$$

konvergiert.

H. Delange [1] zeigte: Sind die multiplikativen Funktionen $f, g$ benachbart, sind beide vom Betrage $\leqslant 1$, existiert der Mittelwert $\mathbb{M}(g)$ und ist

$$
f\left(2^{r}\right)=g\left(2^{r}\right) \quad \text { für alle } r \geqslant 1, \text { falls } g\left(2^{r}\right)=-(-1)^{r} g(2)^{r}
$$$$
\text { für alle } r \geqslant 2 \text { und }|g(2)|=1 \text { gilt, }
$$

so existiert auch $M(f)$.

Die Voraussetzungen dieses Satzes lassen sich abschwächen (man vgl. [10]).

Der Satz von Delange legt folgendes Ergebnis nahe.

SATz 3. Es seien $f$ und $g$ benachbarte multiplikative Funktionen, die folgende Bedingungen erfüllen:

IIit Konstanten $c_{1}>0,0<c_{2}<2, c_{3}>0, c_{4}>0, \delta>0$ gilt:

$$
\sum_{p \leqslant s}\{|f(p)|+|g(p)|\} \leqslant c_{3} \cdot x
$$$$
\left|f\left(p^{r}\right)\right|+\left|g\left(p^{r}\right)\right| \leqslant e_{1} \cdot e_{2}^{r}, \quad \text { für } \quad r=2,3, \ldots ; \text {. }
$$

weiterhin sei

$$
1+\frac{g(p)}{p^{s}}+\frac{g\left(p^{2}\right)}{p^{2 s}}+\ldots \neq 0 \quad \text { in } \quad \text { Res } \geqslant 1
$$

Wenn dann die Reihe

$$
\sum_{n=1}^{\infty} n^{-1} \cdot g(n)=\gamma
$$

(4) Bemerkung. Ist $M(f) \neq 0$ und $M(\bar{g}) \neq 0$, so existiert der Mittelwert $M(f \cdot g)$ auf Grund eines Ergebnisses von. H. Delange [Bull. London Math. Soc. 2 (1970), S. 183-185]. 
konvergiert, so konvergiert auch

(3.4)

$\sum_{n=1}^{\infty} n^{-1} \cdot f(n)=\gamma \cdot \prod_{p}\left(1+\frac{f(p)}{p}+\frac{f\left(p^{2}\right)}{p^{2}}+\ldots\right)\left(1+\frac{g(p)}{p}+\frac{g\left(p^{2}\right)}{p^{2}}+\ldots\right)^{-1}$

gegen den angegebenen Grenzwert.

Beweis. Nach [10] gibt es unter den Voraussetzungen (3.2a) bis (3.2d) von Satz 3 eine multiplikative Funktion $h_{0}$, so da $f=g * h_{0}$ alls Faltung $\left(^{5}\right)$ von $g$ und $h_{0}$ dargestellt werden kann, wobei

$$
\sum_{n=1}^{\infty} n^{-1},\left|n_{0}(n)\right|<\infty
$$

absolut konvergiert. Dann ist

$$
\sum_{n \leqslant N} \frac{f(n)}{n}=\sum_{n \leqslant N} \frac{1}{n} \sum_{d \mid n} h_{0}(d) \cdot g\left(\frac{n}{d}\right)=\sum_{d \leqslant N} \frac{h_{0}(d)}{d} \cdot \sum_{m \leqslant N ! d} \frac{g(m)}{m}
$$

wohlbekannte elementare Abschätzungen zeigen, daß $\sum_{n \leqslant N} n^{-1} \cdot f(n)$ für $N \rightarrow \infty$ gegen $\left\{\sum d^{-1} \cdot h_{0}(d)\right\} \cdot \gamma$ konvergiert, und (5.2) aus [10] gibt die in (3.4) angegebene Produktdarstellung für $\sum d^{-1} \cdot h_{0}(d)$.

FolgerdNG zU SaTz 3. Sei $f \epsilon_{0} \mathscr{F}_{0}$; definient man eine multiplikative Funtition $b: \mathbf{N} \rightarrow \mathbf{C}$ durch

$$
b(n)=\left\{\begin{array}{lll}
\mu^{2}(n) \cdot \prod_{p \nmid n}(1-f(p)) & \text { für } & 2 \nmid n \\
0 & \text { für } & 2\lfloor n
\end{array}\right.
$$

so ist aie Reihe

$$
\sum_{n=1}^{\infty} \frac{b(n)}{n}
$$

konvergent.

Beweis. In [11] wurde gezeigt $\left({ }^{6}\right)$, daß fiur die durch.

$$
v(r)= \begin{cases}\mu^{2}(r) \cdot \prod_{p \mid r}\{1-f(p)\} *\left\{1+\frac{f(p)-1}{p}\right\}^{-1}, & \text { falls }(r, 6)=1 \\ 0, & \text { falls }(r, 6) \neq 1\end{cases}
$$

(5) d.h. es ist $f(n)=\sum_{d \mid n} h_{0}\langle d\rangle \cdot g(n / d)$.

(5) Hierfür waren die verhältnismäßig tief liegenden Hilfsmittel von Halász erforderlich. definierte multiplikative Funktion die Reihe

$$
\sum_{r=1}^{\infty} r^{-1} \cdot v(r)
$$

konvergiert. Offenbar sind die Funktionen $b(\cdot)$ und $v(\cdot)$ benachbart und erfüllen die Voraussetzungen (3.2a) bis (3.2c) von Satz 3; weiterhin ist in Res $s \geqslant 1$

$$
\left|v(p) \cdot p^{-s}\right| \leqslant \frac{2}{p-2}<1 \quad \text { für } p \geqslant \tilde{b}, \quad \text { bzw. }=0 \quad \text { für } p=2,3
$$

somit ist auch (3.2d) erfüllt. Nach Satz 3 konvergiert dann $\sum n^{-1} \cdot b(n)$.

4. Hilfssätze. Nach Delange [1] gilt

LEMMA 1. a) Ist $f: N \rightarrow C$ multiplituativ, $|f| \leqslant 1$, und existiert $M(f) \neq 0$, so konvergient die Reine

und

$$
\sum_{p} \frac{1-f(p)}{p}
$$

$$
\text { 近 } r \geqslant 1 \text {, so daß } f\left(2^{r}\right) \neq-1 \text { ist. }
$$

b) Ist $f: N \rightarrow C$ multiplikativ, $|f| \leqslant 1$ und konvergiert die Reihe (4.1), so existiert der Mittelwert $\left(^{7}\right)$

$$
M(f)=\prod_{p}\left\{\left(1-\frac{1}{p}\right)\left(1+\frac{f(p)}{p}+\frac{f\left(p^{2}\right)}{p^{2}}+\ldots\right)\right\} .
$$

Im weiteren geben wir einige Formeln für die in $\S 2$ definierten Koeffizienten $a_{q}(f)$. Mit (2.1) und (2.2) erhält man leicht

$$
1+\frac{f(p)}{p}+\frac{f\left(p^{2}\right)}{p^{2}}+\ldots=\left(1-\frac{f(p)}{p}\right)^{-1} \cdot \eta(p) .
$$

LEMMA 2. Sei $f \mathscr{F}_{0}$. Wit einer nur von $f$ abhängigen Konstanten $\gamma_{1}$ gilt fiur alle $p$ und alle $r \geqslant 0$ die Abschatzung

$$
\left|a_{p^{r}}^{*}\right| \leqslant \gamma_{1} \cdot(r+1) \cdot p^{-r}
$$

Denn es ist nach (2.3)

$$
\left|a_{p r}^{*}\right| \leqslant \sup _{p}\left|\frac{1}{\eta(p)}\right| \cdot \frac{1}{p^{r-1}(p-1)} \cdot\{4 r+2\} ;
$$

für $\gamma_{1}$ kann man demnach $8 \cdot \sup |1 / \eta(p)|$ nehmen.

(7) Gilt zussätzlich (4.2), so ist $M(f) \neq 0$. 
LEMna 3. Mit der Ablüirzung

$$
e_{p}=\left(1-\frac{1}{p}\right)\left(1+\frac{f(p)}{p}+\frac{f\left(p^{2}\right)}{p^{2}}+\ldots\right)=\left(1-\frac{1}{p}\right)\left(1-\frac{f(p)}{p}\right)^{-1} \cdot \eta(p)
$$

gilt fïr $r=0,1,2, \ldots$ und für $f \in \mathscr{F}_{0}$

$$
e_{p} \cdot\left\{1+a_{p}^{*} \varphi(p)+\ldots+a_{p^{r}}^{*} \varphi\left(p^{r}\right)-a_{p^{r+1}}^{*} \cdot p^{r}\right\}=f\left(p^{r}\right) .
$$

Bemerkung. Dieses Ergebnis findet sich in [9], (3.7), wurde aber dort mit Hilfe einiger Formeln hergeleitet, die hiex nicht zur Verfügung stehen.

Beweis. Mit (2.3) und mit der in (4.6) gegebenen zweiten Darstellung von $e_{y}[$ man $v$ gl. hierzu (4.4)] ist (4.7) fürr $r=0$ und $r=1$ ziemlich rasch nachzurechnen. Eine etwas mühsame vollständige Induktion gibt dann (4.7) für jedes $r$, wobei man noch zweckmäBigerweise die Beziehung

$$
\sum_{0 \leqslant 0 \leqslant r} h\left(p^{e}\right) \cdot f^{r-\rho}(p)=f\left(p^{r}\right), \quad r=0,1,2, \ldots
$$

benützt (die ebenfalls rasch durch vollständige Induktion bewiesen werden kann).

5. Konvergenz der Reihe (2.5). Wie in [11] ist

$$
\sum_{q \leqslant Q} a_{q} c_{q}(n)=\sum_{d \mid n} d \cdot \sum_{r \leqslant Q / d} \mu(r) \cdot a_{r d}
$$

wegen $a_{q}=H(f) \cdot a_{q}^{*}$ genügt es also, für die endlich vielen Teiler $d$ von $n$ die Partialsummen.

$$
s_{d}(R)=M(f) \cdot \sum_{r \leqslant R} \mu(r) \cdot a_{r d}^{*}
$$

für $R \rightarrow \infty$ auf Konvergenz zu untersuchen. Sei $d=\prod 1 p^{\delta}$ die kanonischo Primfaktorzerlegung von $\dot{d}$; wir zerlegen $d=t \cdot D$ in ein Produlkt zweier teilerfremder Falktoren, wobei $\left({ }^{8}\right)$

$$
t=\prod_{p_{\|\| d, a_{p^{\delta}}^{\delta} 00}} p^{\delta}
$$

das Produkt derjenigen Primzahlpotenzen $p^{\delta}$ aus der Faktorzerlegung von $d$ ist, für die $a_{p}^{*} \delta$ verschwindet; demnach ist $a_{D}^{*} \neq 0$.

( $\left.{ }^{8}\right)$ Ein loeres Produkt ist als 1 zu interpretieren.
$\mathrm{Zu} S_{d}(R)$ tragen wegen der Multiplikativität der Funktion $a^{*}$ höchstens solche Terme etwas bei, für die $r \equiv 0 \bmod \alpha(t)$ ist, wobei

$$
\alpha(t)=\prod_{p ! t} p
$$

den quadratfreien Kern von $t$ bezeichnet. Somit erhält man ans (5.1) mit $\gamma=r^{\prime} \cdot a(t)$ und der Embenennung $r^{\prime} \rightarrow r$ wegen der Multiplikativität der Funktion $q \rightarrow a_{q}^{*}$

$$
S_{d}(R)=\mu(\alpha(t)) \cdot a_{t \cdot a(t)}^{*} \cdot M(f) \cdot \sum_{\substack{r \leqslant \frac{R}{a(t)} \\(r, t)=1}} \mu(r) \cdot a_{r D}^{*} .
$$

Die Funktion $r \rightarrow r \cdot B_{i}(r)$ mit

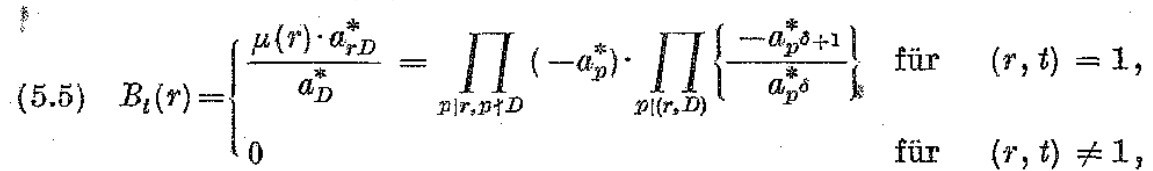
ist multiplikativ und zu der in (3.5) definierten Funktion $b(n)$ benachbart; wegen $(2.3),(3.5)$ und $(5.5)$ ist nämlich $\left({ }^{9}\right)$ fïr $p \nmid 2 \cdot t \cdot D$

$$
\begin{aligned}
p \cdot B(p)-b(p) & =\frac{1}{\eta(p)} \cdot\left\{\frac{p}{p-1}(1-f(p))-p \cdot \sum_{e=1}^{\infty} p^{-\varrho} h\left(p^{\rho}\right)\right\}-\{1-f(p)\} \\
& =\left\{1+O\left(p^{-2}\right)\right\} \cdot\left\{1-f(p)+O\left(p^{-1}\right)\right\}-\{1-f(p)\}=O\left(p^{-1}\right) ;
\end{aligned}
$$

somit konvergiert $\left(^{10}\right)$ die Reihe $\sum_{p} p^{-1} \cdot|p B(p)-b(p)|$. Wir wenden Satz 3 auf die Funktionen $r \rightarrow r \cdot B_{t}(r)$ und $r \rightarrow b(r)$ an; es ist leicht nachzuprüfen (man vgl. $\S 2$ und $\S 4$ ), daß die Voraussetzungen (3.2a), (3.2b) und (3.20) exfüllt sind; schließlich ist für Res $\geqslant 1$

$$
\left|1+\frac{b(p)}{p^{s}}+\frac{b\left(p^{2}\right)}{p^{2 s}}+\ldots\right| \geqslant 1-\frac{2}{p}>0 \text { für } \quad p>2, \quad \text { für } p=2,
$$

und wegen (3.6) und Satz 3 ist

$$
\sum_{r=1}^{\infty} \frac{1}{r} \cdot r B_{i}(r)
$$

konvergent, woraus wegen (5.4) und (5.5) unmittelbar die Konvergenz von $\delta_{d}(R)$ für $R \rightarrow \infty$ und damit die Konvergenz der Reihe $\sum a_{q} e_{q}(n)$ folgt.

(9) Man beachte $h(p)=0,\left|h\left(p^{p}\right)\right| \leqslant 2,|f| \leqslant 1$.

(19) Wegen der Abhängigkeit von $d$ und $d \mid n$ kann gleichmäßige Konvergenz der Reihe $(2.5)$ in $n$ nicht erwartet werden. 
6. Bestimmurng des Grenzwertes der Reihe (2.5). Nach dem Stetigkeitssatz für Diriohletreihen (11) ist der Grenzwert der nach $\S 5$ konvergenten Reihe (2.5) gleich

$$
\lim _{\sigma \rightarrow 0+} A(\sigma, n), \quad A(\sigma, n)=\sum_{q=1}^{\infty} q^{-\sigma} \cdot a_{q} \cdot c_{q}(n) ;
$$

die für $\sigma>0$ absolut konvergente Reihe für $A(\sigma, n)$ besitzt die Produktdarstellung

(6.2)

$$
A(\sigma, n)=M(f) \cdot \prod_{p}\left\{1+\frac{a_{p}^{*} c_{p}(n)}{p^{\sigma}}+\ldots\right\}=\prod_{p}\left\{e_{p} \cdot\left(1+\frac{a_{p}^{*} e_{p}(n)}{p^{\sigma}}+\ldots\right)\right\}
$$

wegen der Produktdarstellung (4.3) für $M(f)$ und mit.(4.6). Man zerlege dieses Produkt in

$$
A(\sigma, n)=\prod_{p \leqslant K}\{\cdots\} \cdot \prod_{p>K}\{\cdots\}=P_{1}(\sigma) \cdot P_{2}(\sigma),
$$

wobei $K$ fest, aber größer als $n$ angenommen wird. Füü das erste Produkt erhält man (fü̈ $K>n$ ).

$$
\lim _{\sigma \rightarrow 0+} P_{1}(\sigma)=P_{1}(0)=f(n),
$$

wie leicht ans Lemma 3 folgt, wenn man beachtet, daß $c_{p^{r}}(n)$ die Werte $\varphi\left(p^{r}\right)$ bzw. $-p^{r-1}$ bzw. 0 annimmt, je nachdem ob $p^{r} \mid n$ bzw. $p^{r-1} \| n$ bzw. $p^{n-1} \nmid n$ gilt. Das zweite Produkt ist von einfacher Bauart; mit (2.3) und (4.6) erhält man für die einzelnen Faktoren von $P_{2}(\sigma)$ die Gestalt

$$
\text { (6.4) } \begin{aligned}
\{\ldots\} & =\frac{p-1}{p-f(p)} \cdot\left\{\eta(p)-\frac{1}{p-1} \cdot \frac{1}{p^{\sigma}}(f(p)-1)-\frac{1}{p^{\sigma}}(\eta(p)-1)\right\} \\
& =\left(1-\frac{1}{p^{\sigma}}\right) \cdot\left\{\frac{p-1}{p-f(p)} \cdot \eta(p)-1\right\}+1 .
\end{aligned}
$$

Ersetzt man $\eta(p)$ nach (2.2) durch $1+O\left(p^{-2}\right)$ und berüleksiehtigt man nur Glieder, die größer als $O\left(p^{-2}\right)$ sind, so folgt für die einzelnen Faktoren von $P_{2}(\sigma)$

$$
\{\ldots\}=\left(1-\frac{1}{p^{\sigma}}\right) \cdot \frac{f(p)-1}{p}+1+o\left(\frac{1}{p^{2}}\right) .
$$

Wegen der Konvergenz der Reihe $\sum p^{-1}(f(p)-1)$ [man vgl. (4.1)] konvergiert (etwa naeh dem 1. Abelsehen Kriterinm $\left({ }^{12}\right)$ ) die Reihe

$$
\sum_{p>\mathbb{E}}\left(\frac{f(p)-1}{p}\right) \cdot\left(1-\frac{1}{p^{\sigma}}\right)
$$

(II) Dieser beruht nur anf einer partiellen Summation.

(12) [6], S. 334 - oder man wende direkt partielle Summation an. gleichmäßig in $0 \leqslant \sigma \leqslant \frac{1}{2}$, also auch das Produkt $P_{2}(\sigma)$, und es ist

$$
\lim _{\sigma \rightarrow 0+} P_{2}(\sigma)=P_{2}(0)=1,
$$

da für $\sigma=0$ nach (6.4) alle Faktoren gleich 1 werden. (6.3) und (6.5) zusammen geben

$$
\lim _{\sigma \rightarrow 0+} A(\sigma, n)=f(n) .
$$

7. Beweis von Satz 2. Wenn $\sum_{q=1}^{\infty}\left|a_{q}\right|^{2} \cdot \varphi(q)$ konvergiert, so ist diese Reihe gleich

$$
=|M(f)|^{\boldsymbol{q}} \cdot \prod_{p}\left(1+\left|a_{p}^{*}\right|^{2} \varphi(p)+\left|a_{p}^{*}\right|^{2} \varphi\left(p^{2}\right)+\ldots\right)
$$

berüeksichtigt man die Produktdarstellung für

$$
\left[\left.M f(f)\right|^{2}=\prod_{p} \mid e_{x}\right]^{2}
$$

[man vgl. (4.3) und (4.6)], so liegt es nahe, die Funktionen

$$
k_{p}(n)=e_{p} \cdot \sum_{r=0}^{\infty} a_{p^{r}}^{*} \cdot c_{p^{r}}(n)
$$

zu betrachten und zanächst für diese die Parseval'sche Gleichung herzuleiten; wegen (4.5) ist die Reibe für $k_{p}(n)$ absolut konvergent $\left({ }^{13}\right)$. Mit $n=p^{\nu} \cdot n^{\prime}, p \nmid n^{\prime}$ und $c_{p^{r}}(n)=\varphi\left(p^{r}\right)$ bzw. $-p^{\nu}$ bzw. 0 für $r \leqslant \nu$ bzw. $r=v+1$ bzw. $r>\nu+1$ and Lemma 3 erhält man sofort

$$
k_{p}(n)=f\left(p^{\nu}\right), \quad \text { wenn } p^{\psi} \| n .
$$

Zur Bestimmang des Mittelwertes von $\left|k_{p}\right|^{2}$ berechnet man

$$
\begin{aligned}
\sum_{n \leqslant x}\left|k_{p}(n)\right|^{2} & =\sum_{0 \leqslant \beta \leqslant \frac{\log x}{\log p}}\left|f\left(p^{\beta}\right)\right|^{2} \cdot \sum_{\substack{n \leq x) p \\
(\boldsymbol{x}, p)=1}} 1 \\
& =x \cdot \frac{\varphi(p)}{p} \cdot \sum_{\beta=0}^{\infty} \frac{\left|f\left(p^{\beta}\right)\right|^{2}}{p^{\beta}} \cdot(1+o(1)),
\end{aligned}
$$

denn es ist

$$
\sum_{x \leqslant y,(n, p)=1} 1=[y]-\left[\frac{y}{p}\right]=y \cdot \frac{\varphi(p)}{p}+O(1)
$$

(13) Die Reihe ist in Wirklichkeit eine endliche Summe, die höchstens bis $\left\lfloor\frac{\log n}{\log p}\right\rfloor+1$ geht. 
Somit ist

$$
M\left(\left|k_{p}\right|^{2}\right\rangle=\frac{\varphi(p)}{p} \cdot \sum_{\beta=0}^{\infty} \frac{\left|f\left(p^{\beta}\right)\right|^{2}}{p^{\beta}}
$$

Andererseits erhält man mit (7.2) und der Abkürzung $R=\left[\frac{\log x}{\log p}\right]+1$

$$
\sum_{n \leqslant x}\left|k_{p}(n)\right|^{2}=\left|e_{p}\right|^{2} \cdot \sum_{0 \leqslant r, r^{\prime} \leqslant R} a_{p^{r}}^{*} \cdot \overline{a_{p^{r}}^{*}} \sum_{n \leqslant x} c_{p^{r}}(n) \cdot a_{p^{r^{r}}}(n) .
$$

Dabei ist die innerste Summe (mit (1.1)) gleich

$$
\begin{aligned}
& S\left(x, r, r^{\prime}\right):=\sum_{n \leqslant x} e_{p^{r}}(n) \cdot c_{p^{r^{\prime}}}(n)
\end{aligned}
$$

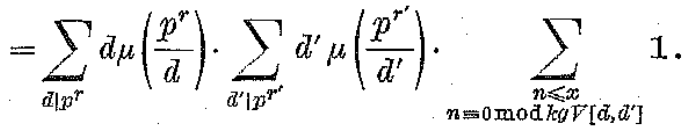

Dies ist leieht auszurechnen, da $d$ nur $p^{r}$ oder $p^{r-1}$ sein kann (entsprechend für $\left.d^{\prime}\right)$; man erhält mit $O$-Konstanten, die von $x, r, r^{\prime}, p$ unabhängig $\operatorname{sind}$,

und

$$
S\left(x, r, r^{\prime}\right)=x \cdot p\left(p^{r}\right)+O\left(p^{2 r}\right) \quad \text { für } \quad r=r^{\prime},
$$

$$
S\left(x, \dot{r}, r^{\prime}\right)=O\left(p^{r+r^{\prime}}\right) \text { füix } r \neq r^{\prime} .
$$

Somit wird, wenn man die Abschätzung (4.6) für $a_{q}^{*}$ beachtet,

$$
\sum_{n \leqslant s}\left|k_{p}(n)\right|^{2}=\left|e_{p}\right|^{2}, \sum_{0 \leqslant r \leqslant R}\left|a_{p^{r}}^{*}\right|^{2} \cdot \varphi\left(p^{r}\right) \cdot x+\left|e_{p}\right|^{2} \cdot \sum_{0 \leqslant r, r^{r} \leqslant R} \sum_{0} \dot{O}(1) ;
$$

wegen $R=O(\log x)$ folgt

$$
M\left(\left|k_{p}\right|^{2}\right)=\left|e_{p}\right|^{2} \cdot \sum_{r=0}^{\infty}\left|a_{p}^{*}\right|^{2} \cdot \varphi\left(p^{r}\right) .
$$

Ein Vergleich mit (7.4) gibt

(7.6) $\quad\left(1-\frac{1}{p}\right) \cdot\left(1+\frac{|f(p)|^{2}}{p}+\frac{\left|f\left(p^{2}\right)\right|^{2}}{p^{2}}+\ldots\right)=\left|e_{p}\right|^{2} \cdot \sum_{r=0}^{\infty}\left|a_{p}^{*}\right|^{2} \cdot p\left(p^{r}\right)$.

Bildet man das Produkt der linken Seite von (7.6) über alle $p$, so konvergiert dieses [man $\mathrm{vgl}$. Lemma $1, b,(4.3)$ ] gegen $M\left(|f|^{2}\right)$. Somit konvergiert auch

$$
\prod_{p}\left\{\left|e_{p}\right|^{2 \cdot} \cdot \sum_{r=0}^{\infty}\left|a_{p^{r}}^{*}\right|^{2} \cdot \varphi\left(p^{r}\right)\right\}
$$

gegen $\mathbb{I}(f(f)$, also konvergiert auch

$$
\sum_{q=1}^{\infty}\left[\left.a_{q}\right|^{2 \cdot} \cdot q(q)=M\left(|f|^{2}\right)\right.
$$

Die Folgerung zu Satz 2 kann lejeht und anf wohlbekannte Weise aus Satz 2 erhalten werden.

\section{Literaturverzeichnis}

1] H. Delange, whur les fonctions arithmétiques matliplicatives, Ann. Seỉent. de l'École Norm. Sup. (3) 78 (1961), S. 273-304.

[2] - On a class of multiplicative arithmetical functions, Scripta Math. 26 (1963), S. 121-141.

[3] M. J. Delsarte, Essai sur l'application de la théorie des fonctions presque périodiques à l'arithmétique, Ann. Seient. de l'École Norm. Sup. (3) 62 (1945), S. $185-204$.

[4] G. Halász, Über die Irittelwerte multiplikativer zahlentheoretischer Funktionen, Acta Math. Acad. Sci. Hung. 19 (1968), S. 365-403.

[5] - Über die Konvergens multiplikativer zahlentheoretiseher Funktionen, Studia Scient. Math. Hung. 4 (1969), S. 171-178.

[6] K. Knopp, Theorie und Anwendung der unendlichen Reihen, Berlin 1922.

[7] S. Ramanujan, On certain trigonometrical sums and their applications in the theory of numbers, Trans. Cambr. Phil. Soc. 22 (1918), S. 259-276.

[8] W. Schwarz, Ramanujan-Entroicklungen stark multiplikativer zahlentheoretiseher Funktionen, Aeta Arith. 22 (1973), S. 329-338.

[9] - Die Ramanujan-Entwicklung reellwertiger multiplikativer Funktionen vom Betrage kleiner oder gleich Eins, Erscheint in J. Reine Angew. Math. 264.

[10] - Eine weitere Bemerking über multiplitkative Funlitionen, 'Coll. Math. 28 (1973), S. 81-89.

[11] - Bananujan-Entwieklungen stark multiplikativer Funktionen, J. Reine Angew. Math. 262/263 (1973), S. 66-73.

[12] A. Wintnex, Eratosthenian averages, Baltimore 1943.

MATHEMATISCHES SEMINAP

DER JOHANN WOLFGANG GOETHE-UNTVERSITÄT

INSTITUT FÜR REINE MAATHEUATIK 